\title{
Assessment of Brain-Dead Potential Organ Donors
}

Jae Myeong Lee*, Su Jung Kim, Won Jung Lee, Eun Jung Park and Young Gi Min

Department of Surgery, Anjou University School of Medicine, Korea

*Corresponding author: Jae Myeong Lee, Department of Surgery, Ajou University School of Medicine, 164 Worldcup-ro, Yeongtong-gu, Suwon 443-380, Korea, Tel: 82-31-219-6025; Fax: 82-31-219-5755; E-mail: ljm3225@hanmail.ne

Received date: Nov 18, 2015, Accepted date: Jan 19 2016, Published date: Jan 26, 2016

Copyright: $\odot 2016$ Lee JM, et al. This is an open-access article distributed under the terms of the Creative Commons Attribution License, which permits unrestricted use, distribution and reproduction in any medium, provided the original author and source are credited.

\begin{abstract}
Introduction: We quantified the potential for organ donation and identified missed opportunities for organ donation in a single institute.

Materials and methods: Data of potential organ donors and the outcome of requests fororgan donation in the intensive care unit (ICU) of Ajou University Hospital between January 2013 and July 2015 were collected and reviewed.

Results: Of 1338 deaths that occurred within 2.5 years in our ICU, there were $189(14.1 \%)$ brain-dead patients, with 150 medically suitable potential organ donors(11.2\%). 116 requests (request rate of $77.3 \%$ ) for organ donation resulted in 50consents (consent rate of $33.3 \%$ ) and 41 transplantations (transplantation rate of $27.3 \%, 3.1 \%$ of total ICU deaths). In 8 cases out of 49 eligible donors, donation failed, because of circulatory death (1 case), proven prostate cancer ( 2 cases), no request from recipient (1 case), poor quality of organs assessed on the operation table ( 2 cases), hidden active tuberculosis (1 case) and hemophagocytic lymphohistiocytosis (1 case).
\end{abstract}

Conclusion: An increase in the organ donation rate may be possible through increasing the number of consents, and through optimizing the identification procedures of potential donors.

Keywords: Tissue and organ procurement; Brain death; Transplantation; Statistics

\section{Abbreviations: \\ ICU: Intensive Care Unit; PMP: Per Million Population}

\section{Introduction}

The Republic of Korea has a low organ donation rate compared with western countries. The 2014 annual organ donation rate in Spain was 36 per million population ( $\mathrm{pmp}$ ) and $21 \mathrm{pmp}$ in the United Kingdom [1]. In the same year, Korea only had $8.6 \mathrm{pmp}$ - its highest value since the year 2000 [2]. A low donation rate can be due to many reasons; a small donor pool, a low consent rate, or high rate of missed organ donors [3].

The number of brain-dead organ donor detections in Ajou University Hospital is the highest in Korea (Figure 1). However, the number of potential and actual donors is still small, the latter being around 20 per year. In order to improve the donation rate, we tried to quantify the maximal possible rate of organ donation and tackled the question of how we can achieve complete detection, complete consent, and optimal medical support of all potential organ donors.

\section{Materials and Methods}

The medical records of deaths occurring in the intensive care unit (ICU) of Ajou University Hospital (Suwon, Korea) from January 2013 to July 2015 were reviewed. Our hospital is a tertiary university hospital with 1090 beds from which 138 are ICU beds (19 in medical
ICU, 16 in general surgical ICU, 20 in emergency ICU, 20 in trauma ICU, 7 in cardiology ICU, 14 in neuro ICU, and 36 in neonatal ICU, 6 in stroke unit). We excluded deaths occurring in the neonatal ICU, because infants under the age of 2 months are not suitable for organ donation.

The brain-dead organ donor management team in Ajou University Hospital consists of two physicians and one coordinator nurse. The two physicians are both intensivists; one is a general surgeon and the other is an emergency medicine doctor. This team is part of Ajou's transplantation center which also coordinates the surgical teams responsible for recipient management.

Data of requests for organ donation, corresponding consents, as well as the outcome of all cases were collected.

\section{Definition of Terms}

1) Brain-dead: The condition of a patient characterized by a Glasgow Coma Scale score of less than 4, coma mentality with irreversible brain damage, and no self-respiration with ventilator care.

2) Medically suitable brain-dead organ donor: A brain-dead patient without absolute contraindication for organ donation. Absolute contraindications are transmittable infections such as active tuberculosis or seropositivity for human immunodeficiency virus, and tumors with malignancy except low grade central nervous system tumors.

3) Request rate: The number of families asked to donate a family member's organs divided by the number of medically suitable braindead organ donors. 
Citation: Jae Myeong Lee, Su Jung Kim, Won Jung Lee, Eun Jung Park, Young Gi Min (2016) Assessment of Brain-Dead Potential Organ Donors. J Transplant Technol Res 6: 154. doi:10.4172/2161-0991.1000154

Page 2 of 6

4) Consent rate: The number of families agreeing to donate divided by the number of medically suitable brain-dead organ donors.

5) Refusal rate: The number of families not agreeing to donate divided by the number families asked.

6) Eligible donor: An organ donor who passed the first brain-death assessment. In Korea, if a donor passes the first brain-death assessment, he or she can be reported to the Korean Network for Organ Sharing, which then starts to arrange the suitable recipients in need.

7) Eligibility: The number of eligible donors divided by the number of medically suitable brain-dead organ donors.

8) Actual donor: An organ donor who underwent organ procurement surgery, including the ones whose organs were not used.

9) Conversion rate: The number of actual donors divided by the number of medically suitable brain-dead organ donors.

10) Transplanted donor: An organ donor who underwent organ procurement surgery, with designated recipients.
11) Transplantation rate: The number of transplanted donors divided by the number of medically suitable brain-dead organ donors.

The definitions of request, consent, and conversion rate are slightly modified versions of those in the report of the Association of Organ Procurement Organizations [4].

\section{Results}

The last decade was characterized by a steady increase of organ donations in the Republic of Korea (Figure 1). While the number of brain-dead organ donors in 2000 was only 52 , this number has climbed to 446 in 2014. The bottom of Figure 1 shows the increase of organ donors detected in Ajou University Hospital. In addition to requests for organ donation from our own patients, we manage brain-dead patients who have been referred to us from other hospitals after consent. In 2012, the number of actual donors managed by Ajou University has been the highest among all hospitals in Korea.

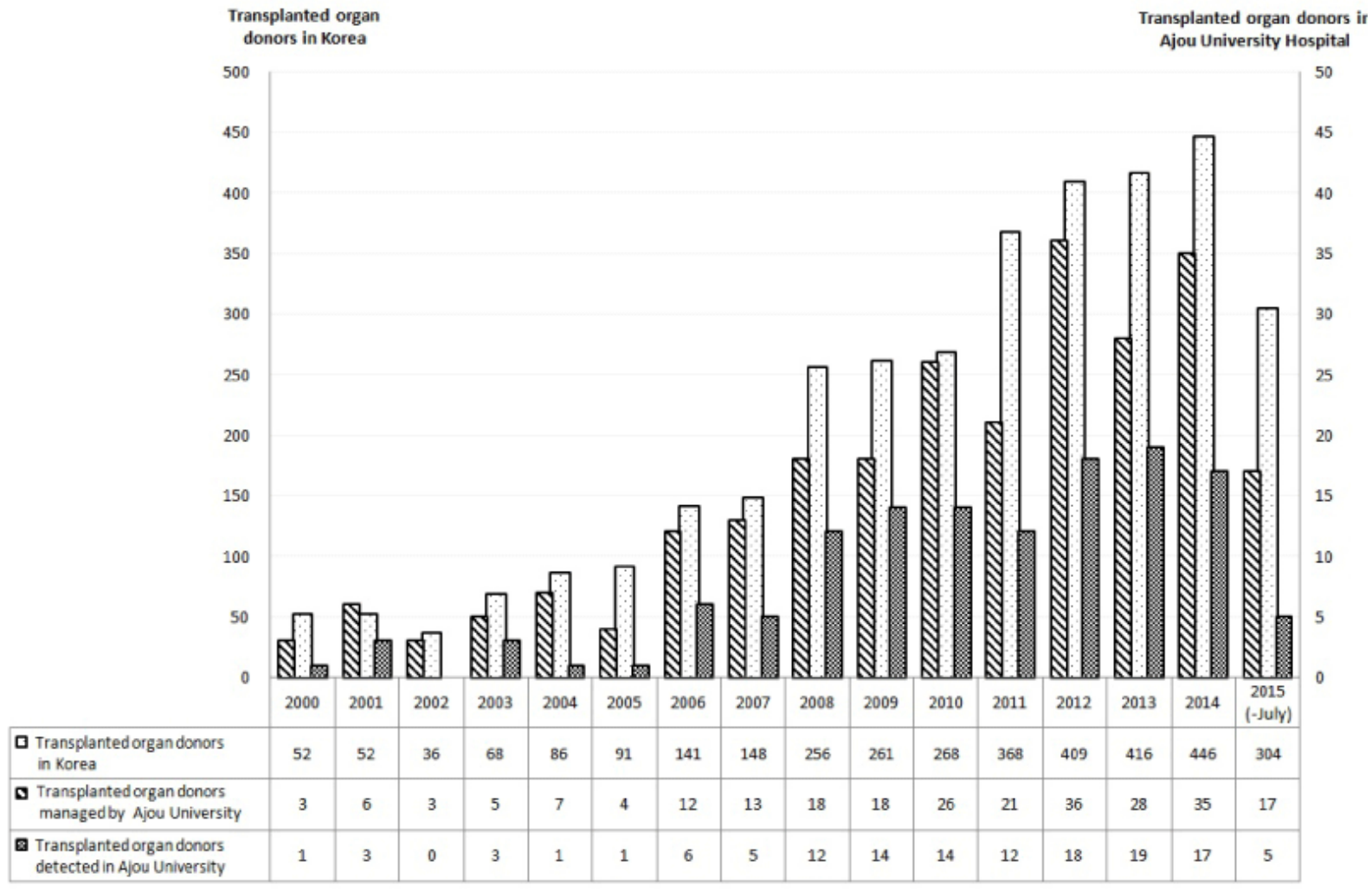

Figure 1: Number of actual organ donations in Korea and Ajou University Hospital from 2000 to July 2015.

Out of 1338 patients who died in the ICU of Ajou University Hospital between January 2013 to July 2015, 189 (14.1\%) were braindead, and $150(11.2 \%)$ were medically suitable brain-dead organ donors (Table 1). 116 requests for organ donation (request rate of $77.3 \%$ ) gave rise to 50 consents (refusal rate of $56.9 \%$ ). From 49 eligible donors (eligibility rate of $32.7 \%$ ), 45 actually donated (conversion rate of $30 \%$ ), and the organs of 41 donors were finally used (transplantation rate of $27.3 \%, 3.1 \%$ of all ICU deaths).

\begin{tabular}{|l|l|l|l|}
\hline & $\mathbf{2 0 1 3}$ & $\mathbf{2 0 1 4}$ & $\begin{array}{l}\mathbf{2 0 1 5} \\
\text { July) }\end{array}$ \\
\hline Intensive care unit deaths & 465 & 528 & 345 \\
\hline Brain deaths & 69 & 82 & 38 \\
\hline Medically suitable brain-dead donors & 51 & 69 & 30 \\
\hline
\end{tabular}


Citation: Jae Myeong Lee, Su Jung Kim, Won Jung Lee, Eun Jung Park, Young Gi Min (2016) Assessment of Brain-Dead Potential Organ Donors. J Transplant Technol Res 6: 154. doi:10.4172/2161-0991.1000154

Page 3 of 6

\begin{tabular}{|l|l|l|l|}
\hline Donation requests & 42 & 52 & 22 \\
\hline Donation consents & 22 & 20 & 8 \\
\hline Eligible donors & 22 & 20 & 7 \\
\hline Actual donors & 21 & 18 & 6 \\
\hline Transplanted donors & 19 & 17 & 5 \\
\hline
\end{tabular}

Figure 3 shows the decision stream from brain-dead patients to transplanted organ donors. The main reason for the rejection of braindead patients as medically suitable donors was hemodynamic instability with multi-organ failure (76.9\%). Family members' nonacceptance of brain death was the most frequent reason why no request for organ donation was undertaken (58.8\%). In 4 cases $(11.8 \%)$, no relatives were available.

Table 1: Data of organ transplantations in Ajou University Hospital.

Figure 2 depicts the different rates for the last 2 and half years. In the course of the decision process leading from medically suitable brain-dead donors to actual donors, high refusal rates (47.6\% [20 out of 42 ] in 2013, 61.5\% [32 out of 52] in 2014, and 63.6\% [14 out of 22] in 2015, Table 1) represent the most important reason for the small number of donors, while low request rates had the second biggest impact. As can be further seen, request and consent rates have been decreasing over the last 2.5 years.

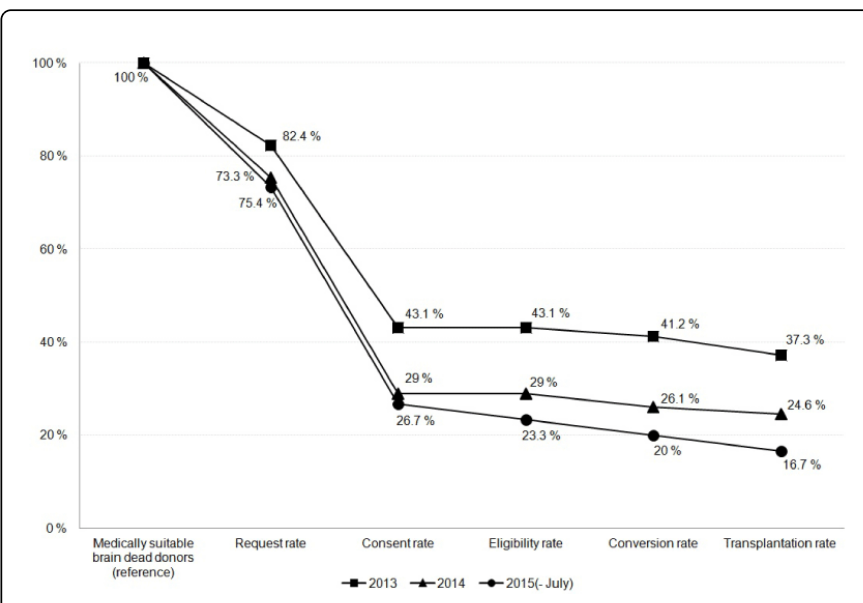

Figure 2: Changes of request, consent, eligibility, conversion, and transplantation rate from January 2013 to July 2015. 
Citation: Jae Myeong Lee, Su Jung Kim, Won Jung Lee, Eun Jung Park, Young Gi Min (2016) Assessment of Brain-Dead Potential Organ Donors. J Transplant Technol Res 6: 154. doi:10.4172/2161-0991.1000154

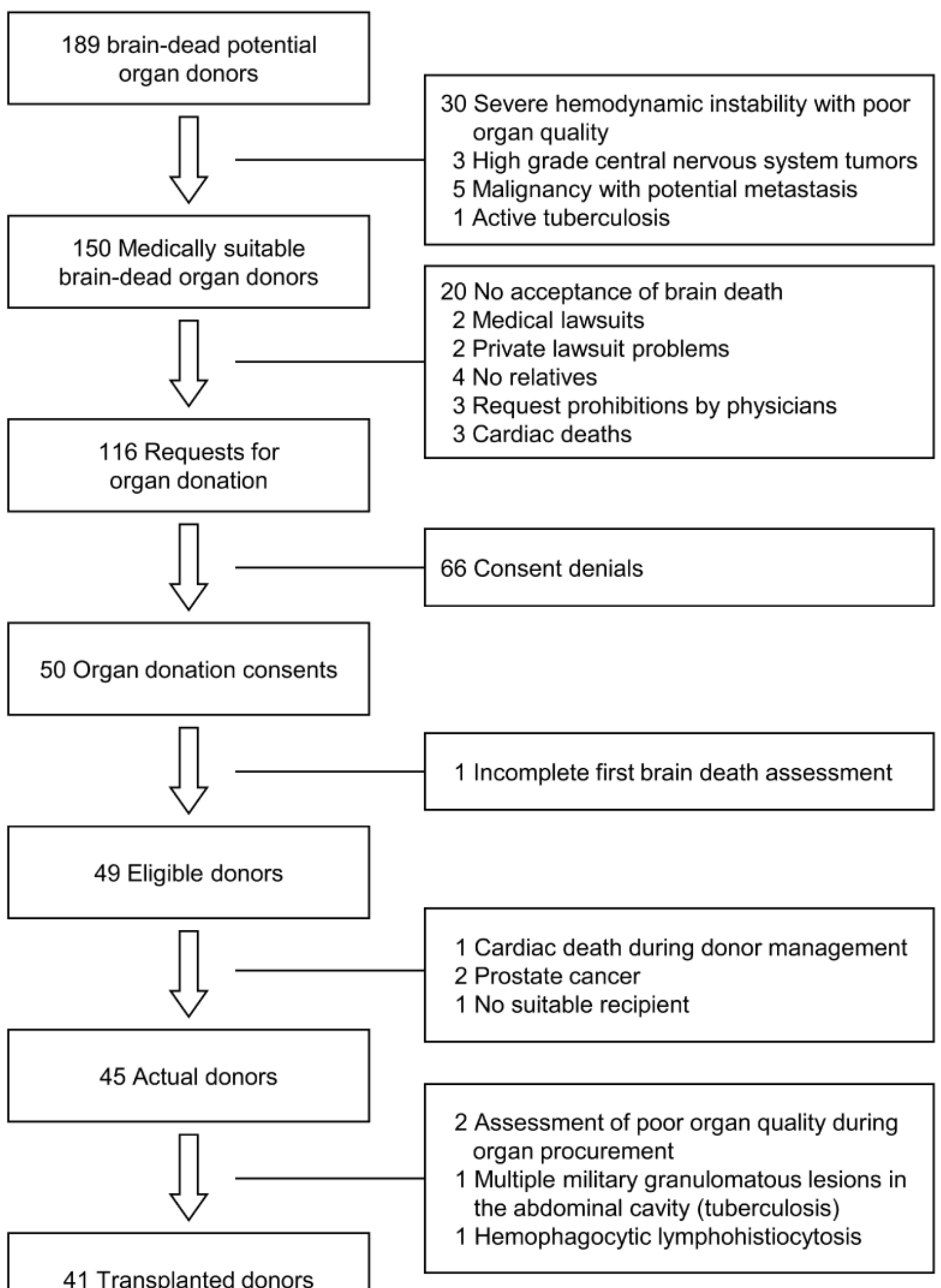

Figure 3: Schematic diagram of the organ donation process from brain-dead potential organ donors to organ transplanted. 
Donation failure from consented donors occurred in 9 cases. One unsatisfactory brain-death assessment prevented eligibility, and one case ended in circulatory death during management. No suitable recipient could be found for one donor with severe hemodynamic instability, even though this patient passed the first brain-death assessment and we looked for recipient candidates in the whole nation. Two cases showed elevated prostate-specific antigens and adenocarcinomas in frozen biopsies of the prostate. Regardless of the cancer stage, we could not proceed to the next step after getting confirmation through biopsies because of the strict Korean law.

At the operation room, 4 donations were discarded. In 2 cases the quality of the organs, which were assessed on the operation table, was so poor that they could not be procured. One case had active tuberculosis in the whole abdomen, and one patient showed hemophagocytic lymphohistio cytosis. A frozen liver biopsy of the latter revealed over $50 \%$ of macrophage phagocytosis.

\section{Discussion}

The rise of Spain's organ donation rate from $14.3 \mathrm{pmp}$ in 1989 to over $30 \mathrm{pmp}$ in 2000 has been in part attributed to strategies including systematic death audits in hospitals and a proactive donor detection program [5]. In the last 10 years, a significant increase in organ donation from both deceased and living occurred also in South Korea. Especially changes in the national systems related to organ donation from deceased could broaden the donor pool [6].

In 2006, the number of donations from deceased in South Korea exceeded 100, and until 2014 the number has increased constantly (Figure 1). This increase is accompanied by rising case numbers of brain-dead organ donors in Ajou University. The total number of donors in our institute includes patients referred from other hospitals, as well as brain deaths in our own institute.

As can be seen in Figure 3, the main reason for the loss of medically suitable organ donors is severe hemodynamic instability. This means that if the managing physicians strengthened their efforts to stabilize the patients, the number of medically suitable brain-dead organ donors might be increased. Whereas malignant tumors or severe infections such as tuberculosis cannot be influenced, keeping the vital signs stable should be possible more frequently.

Only 116 donation requests were made over the last 2.5 years, although 150 medically suitable brain-dead organ donors were available. The most important reason for this relatively low request rate was a bad atmosphere during the notification process of the family members, and their unwillingness to accept the medical condition of brain-death (20 cases, Figure 3).

Another problem was the lack of relatives who could have been asked for donation consent. In 4 cases we were not able not proceed in the donation process because no legal consent could be obtained. In South Korea, physicians should report potential brain-dead donors to the Korean Network for Organ Sharing. However, no law enforces the obligation to notice the condition of brain-death to family members, or to request their permission to transplant usable organs of the braindead patient. It is further prohibited to transplant organs without legal consent from the family. New laws that not only stipulate the duty to request for organ donation, but also allow organ donation in case of non-existence of next kin, are therefore needed.

The most severe drop of potential donors occurred during the consent stage. The average consent rate from Jan. 2013 to Jul. 2015 was a humble $33.3 \%$ (with respect to medically suitable brain-dead organ donors), with a falling tendency (Figure 2). Thirty-six organprocurement organizations in the United States reported consent and conversion rates of $54 \%$ and $42 \%$, respectively, for the years before 2000 [6]. Even though these data are old, the rates are much higher than in our institute. Webb et al. [1] also reported their concern about the low consent rates in the United Kingdom, with a value of only $24.4 \%$ in 2014, due to high refusal rate of organ donation (43\%). They mentioned that the United Kingdom has the second highest refusal rate of organ donation in Europe, even though they still have higher donation rates than Korea [7].

From 50 consented organ donors, 41 were finally transplanted (Figure 3). Although it is hard to prevent these losses, the reasons should be identified and eliminated when possible. The transplantation rate, averaged to only $27.3 \%$ (with respect to medically suitable braindead organ donors), decreased from 37.3\% in 2013 to $16.7 \%$ in 2015.

Because our goal is a successful transplantation with a saved recipient's life, we usually send the donors to the operation table even in cases where the quality of the organs is unclear or can be expected to be relatively poor. Analysis of an organ and judgment whether transplantation is reasonable, is easier if the organ is exposed.

Sometimes we take the risk of failure due to low organ quality because the primary goal is to save or improve the recipients' lives.

In summary, the most important step in order to maximize the number of actual donors is to increase request and consent rates, in line with a previous study from Denmark, which names a high refusal rate (about 50\%) as the most important reason for failure [6,8-12]. Donation cconsent is more likely if the request is made by organ donation experts who are well informed about backgrounds, procedures, and benefits of organ donation [3].

It requires an appropriate and careful approach to the family members of the potential donor. This approach should be done in cooperation with an organ donor management team, as well as with an organ-donation society. The Korean society's cultural and emotional change of perception about organ donations from brain-dead patients is inevitable if the situation is to be improved. The greatest opportunity for increasing the rate of donation from the pool of brain-dead potential donors lies in increasing the positive perception about brain dead organ donation and consent rate.

\section{Conclusion}

Small consent rates are the primary cause for the discrepancy between the number of potential organ donors and the number of actual organ donations. Identification of potential organ donors and increasing request and consent rates are the most effective measures to boost organ donations. New laws that enforce donation request in case of brain-death should be considered.

\section{References}

1. Webb G, Phillips N, Reddiford S, Neuberger J (2015) Factors Affecting the Decision to Grant Consent for Organ Donation: A Survey of Adults in England. Transplantation 99: 1396-1402.

2. Korea Organ Donation Agency (2014) KODA Annual Report Seoul: Korea Organ Donation Agency.

3. Opdam HI, Silvester W (2004) Identifying the potential organ donor: an audit of hospital deaths. Intensive Care Med 30: 1390-1397. 
Citation: Jae Myeong Lee, Su Jung Kim, Won Jung Lee, Eun Jung Park, Young Gi Min (2016) Assessment of Brain-Dead Potential Organ Donors. J Transplant Technol Res 6: 154. doi:10.4172/2161-0991.1000154

Page 6 of 6

4. Sheehy E, Conrad SL, Brigham LE, Luskin R, Weber P, et al. (2003) Estimating the number of potential organ donors in the United States. N Engl J Med 349: 667-674.

5. Matesanz R, Miranda B (2002) A decade of continuous improvement in cadaveric organ donation: the Spanish model. J Nephrol 15: 22-28.

6. Min SI, Ahn C, Han DJ, Kim SI, Chung SY, et al. (2015) To achieve national self-sufficiency: recent progresses in deceased donation in Korea. Transplantation 99: 765-770.

7. International Registry in Organ Donation and Transplantation (IDROaT).

8. Madsen M, Bogh L (2005) Estimating the organ donor potential in Denmark: a prospective analysis of deaths in intensive care units in northern Denmark. Transplant Proc 37: 3258-3259.
9. Gortmaker SL, Beasley CL, Sheehy E, Lucas BA, Brigham LE, et al. (1998) Improving the request process to increase family consent for organ donation. J Transpl Coord 8: 210-217.

10. DeJong W, Franz HG, Wolfe SM, Nathan H, Payne D, et al. (1998) Requesting organ donation: an interview study of donor and nondonor families. Am J Crit Care 7: 13-23.

11. Siminoff LA, Gordon N, Hewlett J, Arnold RM (2001) Factors influencing families' consent for donation of solid organs for transplantation. JAMA 286: 71-77.

12. Simpkin AL, Robertson LC, Barber VS, Young JD, Shafer (2009) Modifiable factors influencing relatives' decision to offer organ donation: systematic review. 338: 991. 